\title{
Diagonal-SPRITE and Its Applications for In Vivo Imaging at High Field
}

\author{
Andrea Protti ${ }^{1}$, Amy Herlihy ${ }^{2}$, Jean Tessier ${ }^{3}$, Po-Wah So ${ }^{2}$, Tammy Kalber ${ }^{1}$ and Jimmy D. Bell*,1 \\ ${ }^{1}$ Metabolic and Molecular Imaging Group; ${ }^{2}$ Biological Imaging Centre, Imaging Sciences Department, MRC Clinical \\ Sciences Centre, Imperial College London, Hammersmith Hospital Campus, London, UK; ${ }^{3}$ AstraZeneca, Alderley Park, \\ Mereside, Macclesfield, UK
}

\begin{abstract}
Many solid or semi-solid tissues have ultra-short or short T2* components that make them virtually "invisible" to standard MRI techniques. For this reason, methods such as ultra-short echo time (UTE) techniques were developed to investigate and detect some of these short and ultra-short time components. Here we report on the development and implementation of a novel version of the Single Point Ramped Imaging with T1 Enhancement (SPRITE) technique in order to image mouse models. The method, called diagonal-SPRITE, was optimized within hardware limitations such as duty cycle and gradient strength at high field. Preliminary in vivo MR T2* value of organs of normal mouse models and related $\mathrm{T} 2 *$ maps were generated. In addition, the sequence was applied to highlight stomach short $\mathrm{T} 2 *$ after the application of a long T2* suppression pulse and to achieve a positive contrast in a SPIO-labelled cells experiment.
\end{abstract}

\section{INTRODUCTION}

Magnetic Resonance Imaging (MRI) is a powerful imaging modality for in vivo imaging of soft tissue, providing anatomical, functional, and biochemical information as well as unique high spatial resolution. However, standard MRI techniques do not provide good images of tissues with short and ultra-short $\mathrm{T} 2 *$ such as tendon or harder tissues such as bone. To investigate biological tissues with a $\mathrm{T}^{*}$ shorter than $1 \mathrm{~ms}$, ultra-short echo time (UTE) techniques have been developed [1]. Several disease states are likely to benefit significantly from UTE imaging techniques, including monitoring the development of fibrotic tissue in the liver, muscle and lungs, but also in the localization of superparamagnetic iron particles (SPIO) in cell tracking experiments.

Three types of UTE techniques are currently being developed. Recently a method known as sweep imaging with Fourier transformation (SWIFT) [2,3] has been prepared as an alternative to more robust radial projection reconstruction (PR) method [4,5]. The radial technique, initially developed for lung parenchyma imaging [6,7], has also been used to investigate atherosclerosis [8], boron-11 imaging [9] and many biological tissues such as bones [10]. A further method is based on Single Point Imaging (SPI), or Single Point Ramped Imaging with T1 Enhancement (SPRITE) techniques [11,12]. The SPRITE technique developed out of the solids NMR field, where research is being done into imaging solid materials such as Teflon tubing and concrete samples of different composition [13,14]. More recently it was applied in the clinic for sodium imaging of the brain [15]. In preclinical studies, an in vivo SPI work was carried out on lungs at $4.7 \mathrm{~T} \mathrm{[16].}$

In this paper we focus on the SPRITE method. SPRITE has the advantage of being a single point imaging method with no frequency encoding window; it is therefore free from

*Address correspondence to this author at the Molecular Imaging Group, MRC Clinical Sciences Centre, Imperial College London, Hammersmith Hospital Campus, London, UK; E-mail: jimmy.bell@csc.mrc.ac.uk image distortions due to chemical shift and susceptibility at ultra-short acquisition times [17]. Because SPRITE is a pure phase encode technique, it is intrinsically a volume technique, requiring methods such as sensitivity of the RF coil or saturation bands to limit the field-of-view (FOV). It should also be noted that scan times are generally long in order to achieve high resolution. As the FOV and data acquisition time are directly related to the performance of the scanning system, gradient coil specifications (maximum strength and duty cycle) can also be a limiting factor. This represents a limit when SPRITE is used in the clinical setting as reported in the conical-SPRITE case [15] where ultrashort $\mathrm{T} 2 *$ images were obtained in $30 \mathrm{~min}$ at low resolution. No clear anatomical details could be observed. On the contrary, in pre-clinical high field system, due to the use of higher gradients strength, SPRITE can achieve good spatial resolution and anatomical details are distinguishable.

As preclinical work moves to higher field strengths to achieve better SNR, new challenges are emerging. These include: distortions due to Bo field inhomogeneities, susceptibility, chemical shift and eddy current artefacts. Some of the artefacts may be partly overcome by using single point imaging techniques such as SPRITE. However, the need to scan relatively small objects (mouse organs) and the need for good resolution together with existing hardware limitations, have limited the first proposed SPRITE method of Balcom et al. [12]. In order to work well within hardware limitations, a variant of the conventional SPRITE was created. Such technique makes maximum use of the gradient coil strength and duty cycle of a given system. The technique named $d i$ agonal-SPRITE utilizes a diagonal k-space trajectory.

To demonstrate the value of this novel SPRITE approach we have used the method in three ways. First to determine T2* maps of mouse tissues in vivo. Second, it was used to achieve positive contrast from SPIO-labelled cells injected into a hind leg of in vivo mice in order to differentiate the SPIO site from MRI signal voids and blood. Third, diagonalSPRITE was applied with a T2* long suppression pulse in 
front in order to highlight short $\mathrm{T} 2 *$ components in stomach and liver.

\section{THEORY}

\section{Pulse Sequence Development Section}

The original SPRITE trajectory suggested by Balcom et al. [12] traverses k-space on a Cartesian grid. This is achieved by stepping one of the gradients through $\mathrm{k}$-space in discrete steps while the other two gradients are held constant. The three phase encoding gradients position a point in a $3 \mathrm{D}$ time domain matrix. SPRITE has limited spatial resolution because it quickly runs up against gradient duty cycle limitations. On our gradient set, which has a strength of $20 \mathrm{G} / \mathrm{cm}$ and a $9 \%$ duty cycle, it was found that this is primarily due to exceeding the duty cycle limit from one of the constant gradients. In order to overcome this problem a novel hardware efficient k-space trajectory was developed.

A diagonal k-space trajectory was chosen and hence the name: diagonal-SPRITE (Fig. 1A). As for the original SPRITE method, diagonal-SPRITE reconstruction adopts no $\mathrm{k}$-space regridding. The diagonal trajectory samples along a Cartesian grid. In fact, if diagonal-SPRITE is to cover a matrix of $128 \times 128$ pixels, the number of applied RF pulses and consequently the number of acquired points will be
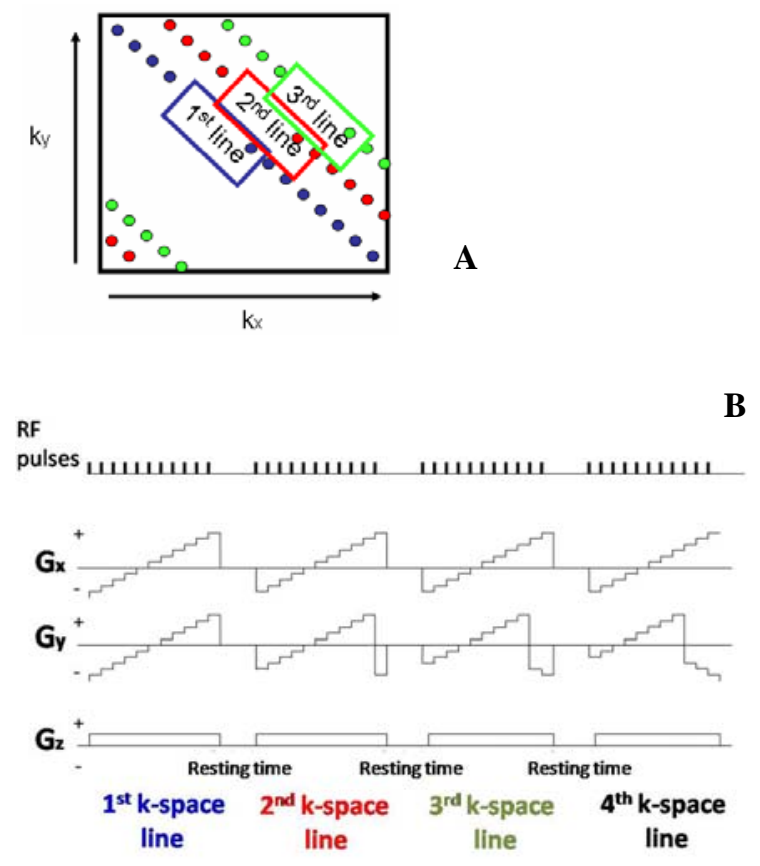

Fig. (1). A) Diagonal-SPRITE k-space acquisition. The acquisition follows a diagonal trajectory. B) Diagonal-Sprite pulse sequence. Stepping the gradients in two of the three Cartesian directions (usually $\mathrm{X}$ and $\mathrm{Y}$ ) $\mathrm{k}$-space is collected upon a diagonal trajectory. A constant gradient on the third imaging axis generates the acquisition of a 2 dimensional image. The gradient is maintained constant along one entire slice. To generate a 3D image volume, the third gradient is stepped in a standard raster method. In the picture above the third gradient is shown for one slice only. The FID points are collected at phase encode time $\left(\mathrm{T}_{\mathrm{p}}\right)$ after a $2^{\circ} 4 \mu$ s in length hard RF pulse. A line of k-space is collected without switching off the gradients. However, there remains a resting time between the lines.
$128 \times 128=16384$. Diagonal-SPRITE is acquiring the entire kspace maintaining the characteristics of a single point imaging technique without an undersampling approach. Thus, image reconstruction can be generated by simply Fourier transforming k-space.

The diagonal trajectory steps two gradients dynamically at all times, while the gradient applied in the third direction is stepped, as for the original SPRITE, in a standard raster method (Fig. 1B).

Diagonal-SPRITE makes better use of gradient duty cycle when compared to SPRITE because none of the gradients are switched on for a long time at maximum strength unless an image volume of hundreds of slices is required. Therefore, there is a reduced demand on the hardware and as a result the resting time can be shorten. A resting time is left between detection of lines of $\mathrm{k}$-space, in order to not exceed gradient duty cycle limits.

For large number (hundreds) of slices, the third gradient, which is usually run at low strength, may become large enough for its duty cycle to become the limiting factor, a situation which can be mitigated using longer resting time.

\section{Signal Equation and T1 Effects}

The signal equation for a steady-state technique such as SPRITE is generally defined as:

$S\left(t=\mathrm{T}_{\mathrm{p}}\right)=M_{0} e^{-t / T 2^{*}} \frac{1-e^{-T R / T 1}}{1-\cos \alpha e^{-T R / T 1}} \sin \alpha$

where $T_{p}$ is the phase encode time, TR is the time period between successive RF excitation pulses, $\alpha$ is the flip angle and $\mathrm{M}_{0}$ is the equilibrium longitudinal magnetization. This signal equation is valid for a coherent steady-state technique. The equation can remove the T1 dependency term in two cases: when the flip angle $\alpha$ is small or when TR is long (of the same order of T1).

In the first case (small flip angles) where the flip angle is set to $2^{\circ}$ or lower with a pulse length of $4 \mu \mathrm{s}$, Eq. (1) turns into a $\mathrm{T} 2 *$ dependent signal equation as follows:

$S\left(t=\mathrm{T}_{\mathrm{p}}\right)=M_{0} e^{-t / T 2^{*}} \sin \alpha$

In the second case (long TR), if TR is long enough to accomplish the full magnetization recovery after excitation, every single k-space point can be related to the longitudinal magnetization at the equilibrium value $\mathrm{M}_{0}$ and therefore to Eq. (2). Long TR, used by SPI techniques, cannot be used by SPRITE techniques especially when biological samples are under study, because of long scan time issues.

Small flip angles have the added advantage of avoiding T1 blurring effects. This effect was mentioned by Mastikhin et al. [18]. From such study the maximum number of points $\left(\mathrm{N}_{\max }\right)$ sampled during acquisition of SPRITE in one direction was therefore defined by:

$N_{\max }<\frac{\pi}{\left(T R / T_{1}-\ln (\cos \alpha)\right)}$

Eq. (3) estimates the limit where a dynamic approach to steady state begins to decrease resolution. When diagonalSPRITE is run at high magnetic field on biological tissues 
with an average T1 of $1500 \mathrm{~ms}$, a flip angle of $2^{\circ}$ and a TR of $1 \mathrm{msec}, \mathrm{N}_{\max }$ approaches 2000. Any matrix dimension less than $\mathrm{N}_{\max }$ ensures that no T1-effects are introduced into the image.

\section{Multi- $\mathbf{T}_{\mathrm{p}}$ Acquisition}

In order to increase the volume efficiency of diagonalSPRITE, we developed and implemented a multi- $T_{p}$ acquisition. This is not the traditional multiple point acquisition where consecutive points are collected on the same FID at longer time phase. In the technique here described, diagonalSPRITE applies as many $2^{\circ} 4 \mu \mathrm{s}$ RF pulses as needed to collect an entire line of $\mathrm{k}$-space at a given $\mathrm{T}_{\mathrm{p}}$ (for instance 0.3 $\mathrm{ms}$ ) and at a longer $T_{p}$. Such scheme acquires the same kspace line at a different $T_{p}$ with no dead time between the two acquired lines (Fig. 2). The process can be applied at more than two different $\mathrm{T}_{\mathrm{p}} \mathrm{s}$.

The resting time is dictated by various factors: the maximum gradient strength used, the gradients duration, the gradient duty cycle. This method gives a reduction in temporal resolution when compare with single diagonal-SPRITE acquisitions.



Fig. (2). diagonal-SPRITE multi-Tp acquisition approach. After a short resting time the same lines of $\mathrm{k}$-space is detected at two different $T_{p}$ (in this diagram only two $T_{p}$ have been used $T_{p}=0.3 \mathrm{~ms}$ and $T_{p}=2 \mathrm{~ms}$ ). The process is repeated for all the $\mathrm{k}$-space lines.

In multi- $T_{p}$ acquisitions, images at different phase encode time are achieved without changing spatial resolution. The gradient strength is adjusted for each $T_{p}$ as dictated by the following equation [11]

Gradientstep $_{x, y, z}=\frac{1}{\gamma * \mathrm{~T}_{\mathrm{p}} * F O V_{x, y, z}}$

where $\gamma$ is the gyromagnetic ratio. At a fixed FOV and a changeable $T_{p}$, the modified parameter is the gradient strength $G$ on each of the three axes.

Multi- $T_{p}$ method is time efficient when compared to single diagonal-SPRITE acquisitions. For instance, if acquisitions, one at ultra-short $\mathrm{T}_{\mathrm{p}}(0.3 \mathrm{~ms})$ and the other at longer $\mathrm{T}_{\mathrm{p}}$ ( $2 \mathrm{~ms}$ ), are run separately, the total acquisition time can rise by $30 \%$ when compare with a multi- $\mathrm{T}_{\mathrm{p}}$ scheme. This is due to the reduction of the resting time between a multi- $T_{p}$ scheme and single acquisitions scheme. A single ultra-short acquisition at a $T_{p}$ of $0.3 \mathrm{~ms}$ requires a resting time of 600 $\mathrm{ms}$ between detected lines of $\mathrm{k}$-space when the maximum gradient strength on our scanner is used. Further, a $2 \mathrm{~ms} T_{p}$ acquisition requires a resting time of $100 \mathrm{~ms}$. On the contrary, the multi- $T_{p}$ scheme requires only a small resting time, usually $100 \mathrm{~ms}$, due to the optimized use of gradient strength.
The image reconstruction for a multi- $T_{p}$ acquisition is identical to the reconstruction for a single $T_{p}$ volume. The $k$ space data acquired from the multi- $\mathrm{T}_{\mathrm{p}}$ scan, are separated according to the $T_{p}$ data set which they belong. For both $T_{p} s$, as no under-sampling is used, the data fill up the entire kspace. In addition, because of the Cartesian diagonal trajectory, no regridding is necessary before a Fourier transformation is used to complete the image reconstruction.

\section{T2* Suppression Pulse}

$\mathrm{T} 2 *$ suppression pulses are based on the observation that it is less demanding to excite long $\mathrm{T} 2 *$ species than to excite short T2* species [19]. Short T2* species are not affected by low-amplitude, long-duration pulses because their transverse magnetization decays faster than it is excited. As $\mathrm{T}_{2} *$ increases, the excitation rate dominates the transverse relaxation. Thus, long-T2* species are fully excited and short-T2* species are relatively unaffected.

In terms of spectral linewidths, $\mathrm{T} 2 *$ is inversely proportional to the linewidth, meaning that short $\mathrm{T} 2 *$ species have broad linewidths and long $\mathrm{T} 2 *$ species have narrow linewidths. The narrow spectrum of a long $\mathrm{T} 2 *$ species is more easily covered by the RF spectrum and thus is easily excited. The broad short $\mathrm{T} 2 *$ species spectrum requires a wide bandwidth RF pulse to be excited. A narrow bandwidth RF pulse will fully excite the longer $\mathrm{T} 2 *$ species, but only partially excite the shorter $\mathrm{T} 2 *$ species, making it an effective long-T2* suppression pulse.

The pulse used in this study is a selective $90^{\circ}$ pulse acting as a band selective pulse. The pulse was designed as a sinc function $(\sin (\mathrm{x}) / \mathrm{x})$ and presented the same characteristics as a $\pi / 2$ pulse. It excited a narrow linewidth of the entire spectrum which corresponded to long $\mathrm{T} 2 *$ components when the pulse was applied to proton resonance. The pulse was applied between 5 and $8 \mathrm{~ms}, \mathrm{~T} 2 *$ species longer than such duration appeared well suppressed. Dephasers were placed before and after the $\mathrm{T} 2 *$ suppression pulse. While long $\mathrm{T} 2 *$ species were excited and dephased, the short $\mathrm{T} 2 *$ had time to decay and thus to restore the magnetization along Bo.

Off-resonance frequency signals, such as that of fat, were not suppressed.

\section{METHOD}

\section{In Vivo Scanning}

Scans were carried out on a 9.4T horizontal bore Varian Inova scanner (Palo Alto, CA) using a $25 \mathrm{~mm}$ ID birdcage RF coil (Magnetic Laboratories, Oxford UK), gradient strength $200 \mathrm{mT} / \mathrm{m}, 9 \%$ duty cycle. All in vivo procedures were conducted in accordance with the UK Animals (Scientific Procedures) Act 1986. Anesthesia was maintained at $1.5 \%$ isoflurane/oxygen mix and body temperature was maintained using warm air (SA Instruments Stony Brook $\mathrm{NY}$ ). None of the in vivo experiments were respiratory or ECG gated.

In the first experiment, a dead mouse was used to compare diagonal-SPRITE and SPRITE in terms of resolution and acquisition time. The acquisition time (15min 16sec) was maintained the same for the two experiments. The field of view varied such that for SPRITE it was 70x70x30 m and 
for diagonal-SPRITE was $30 \times 30 \times 30 \mathrm{~m}$. The following parameters were used for both scans: TR was $0.9 \mathrm{~ms}(0.6 \mathrm{~ms}+$ $\mathrm{T}_{\mathrm{p}}$ (phased encode time)); $\mathrm{T}_{\mathrm{p}}$ was $0.3 \mathrm{~ms}$ and the resting time set to $250 \mathrm{~ms}$. The matrix size was $120 \times 120 \times 21$ and flip angle of $2^{\circ} 4 \mu$ s. Duty cycle limit was not exceeded either in SPRITE or diagonal-SPRITE with such parameters. However, duty cycle values for the two techniques were estimated to approach the $9 \%$ of maximum duty cycle of the 9.4T scanner.

Diagonal-SPRITE was then applied to measure T2* of pulmonary vessels, stomach, spleen and gut in vivo experiments for normal mice. The FOV was maintained at $30 \times 30 \times 30 \mathrm{~m}$, matrix size $120 \times 120 \times 21$. TR was $0.6 \mathrm{~ms}+$ $\mathrm{T}_{\mathrm{p}}$ and the resting time was set to $100 \mathrm{~ms}$. $0.6 \mathrm{~ms}$ was the time required for gradient stabilization in order to avoid eddy current and gradient switching transient effects which might cause image distortion. In order to increase time efficiency a multi- $T_{p}$ acquisition technique was used. For measuring the $\mathrm{T} 2 *$ of stomach and spleen, $\mathrm{T}_{\mathrm{p}}$ were: $0.3,0.7,1,2.5,3.1 \mathrm{~ms}$, acquisition time of $63 \mathrm{~min}$. For gut, $\mathrm{T}_{\mathrm{pS}}$ were set to: $0.3,0.8$, $1.5,2.5,3.8 \mathrm{~ms}$, acquisition time of $70 \mathrm{~min}$. For lungs, $\mathrm{T}_{\mathrm{ps}}$ were: $0.3,0.5,1,1.5 \mathrm{~ms}$, acquisition time of $38 \mathrm{~min}$. Every organ was imaged on 3 different normal mice.

The long $\mathrm{T} 2 *$ suppression pulse experiment was carried out on stomach and liver, where diagonal-SPRITE was run at an ultra-short $\mathrm{T}_{\mathrm{p}}$ of $0.3 \mathrm{~ms}$ with and without pulse preparation. The pulse was applied for $8 \mathrm{~ms}$ at a power of $40.9 \mathrm{~dB}$ and flip angle of $150^{\circ}$.

The parameters for the two experiments were: $T_{p} 0.3 \mathrm{~ms}$, resting time $200 \mathrm{~ms}$, matrix 120x120x21, slice thickness 1.4 $\mathrm{mm}$, flip angle $1.6^{\circ} 4 \mu \mathrm{s}$. The acquisition time was $13 \mathrm{~min}$ and 8 min for diagonal-SPRITE with T2* suppression pulse and diagonal-SPRITE without suppression pulse, respectively.

In the in vivo SPIO-labelled cells experiment, 4 mice (Balb/c, female, Harlan U. K.) were injected with biotinylated cells labelled with anti-biotin SPIO $\left(2 \times 10^{6}, 20 \mu \mathrm{l}\right.$ PBS $)$ into the hind leg and control biotinylated cells (non-SPIO labelled cells) into the contralateral hind leg. DiagonalSPRITE used such scanning parameters: FOV 30×30×30 m,
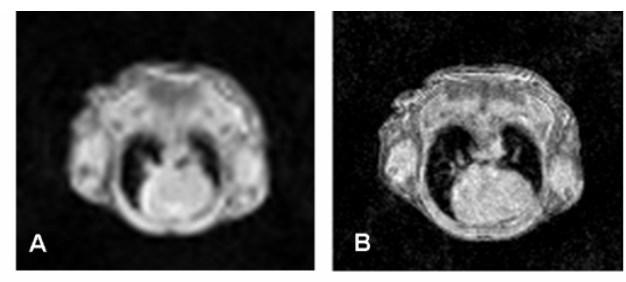

Fig. (3). A) Transverse image of heart of a dead mouse by the use of standard-SPRITE and B) diagonal-SPRITE. The two methods used an acquisition of $15 \mathrm{~min} 16 \mathrm{sec}$. The same scanning parameter (TR, $T_{p}$, flip angle and slice thickness of $1 \mathrm{~mm}$ ) were used for both standard and diagonal-SPRITE. However, due to duty cycle limitations, the minimum FOV used for standard-SPRITE acquisition was $70 \times 70 \times 30 \mathrm{~m}$ while in diagonal-SPRITE case it was reduced to 30x30x30 m with visible resolution changes.
1 average, flip angle of $2^{\circ}$, matrix was $120 \times 120 \times 21$, slice thickness $1.5 \mathrm{~mm}$, resting time $100 \mathrm{~ms}$ and $\mathrm{T}_{\mathrm{ps}} 0.25,0.7,1.4$, $2.8,3.5 \mathrm{~ms}$. Total scan time of 80 minutes. A subtraction method was used to highlight the short T2* of SPIO-labelled cells.

\section{RESULTS}

Axial images of the dead mouse with different resolution (0.58 $\mathrm{mm}$ in Fig. (3A), $0.25 \mathrm{~mm}$ Fig. (3B)) were obtained using the original SPRITE technique and diagonal-SPRITE. This difference in resolution is due to the efficiency of diagonal k-space trajectory compared with standard raster trajectory in scanning the same sample for the same length of time. The lung cavity, the heart and muscle can be observed in both images, although tissue details are better identified in Fig. (3B).

An example of transverse in vivo images through the abdomen obtained with diagonal-SPRITE is shown in Fig. (4). Gut (indicated by the full arrow), part of intestine (dotted arrow), a section of spinal cord (dashed arrow) and muscle are visible. The series of images, of the same slice with 5 different $T_{p}$ acquisitions, emphasizes the shortening in signal intensity of gut. The $\mathrm{T} 2 *$ of this tissue corresponded to 1.16 $\pm 0.24 \mathrm{~ms}$. The same behavior was found in pulmonary vessels, stomach and spleen (images not shown). The exponential decay of pulmonary vessels reported a T2* of $0.35 \pm$ $0.03 \mathrm{~ms}$. The $\mathrm{T}^{*}$ decay of spleen was found to be as short as $2.07 \pm 0.15 \mathrm{~ms}$. The $\mathrm{T} 2 *$ related to stomach was $2.7 \pm 0.3$ $\mathrm{ms}$.

The $\mathrm{T} 2 *$ decay of stomach and gut appears to be dependent on the type of food used to feed the mice. In this case standard rodent chow was used. In addition, it has to be noted that the background noise observed in Fig. (4) increases due to the increasing motion experienced at $T_{p}$ longer than $3 \mathrm{~ms}$ compared to that at ultra-short $\mathrm{T}_{\mathrm{p}}$. Susceptibility effects start to show up in images acquired with $T_{p}$ s longer than $1 \mathrm{~ms}$ as can be seen around the gut. In addition, spin dephasing effects are more pronounced at long $\mathrm{T}_{\mathrm{p}} \mathrm{s}$.

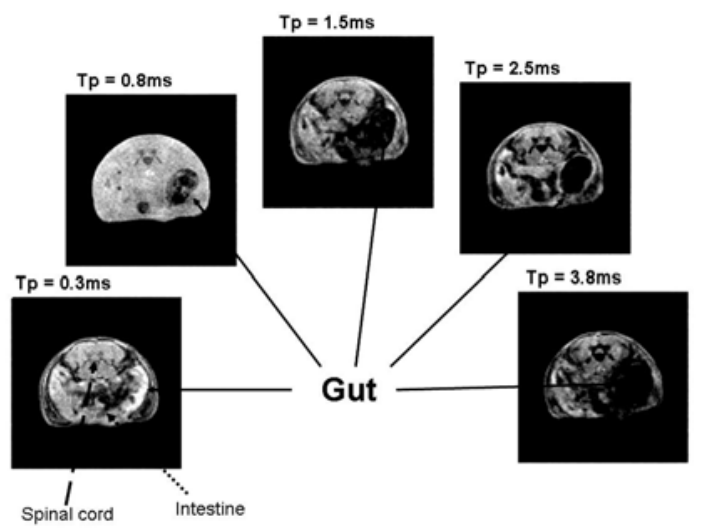

Fig. (4). Gut (full arrow) signal decay for the $5 \mathrm{~T}_{\mathrm{p}}$ acquired by $d i$ agonal-SPRITE. From a high signal intensity at ultra-short $\mathrm{T}_{\mathrm{p}}$, the signal decays to the level of background noise for $T_{p}$ of $3.8 \mathrm{~ms}$. Spinal cord (dashed arrow) and intestine (dotted arrow) are also shown. The same slice is reported for the $5 \mathrm{~T}_{\mathrm{p}}$ acquisitions.

Fig. (5) shows a typical T2* map through the mouse abdomen. The bright areas identify structure with ultra-short 
and short $\mathrm{T} 2 *$ components such as gut or bones, while dark areas identify the long $\mathrm{T} 2 *$.

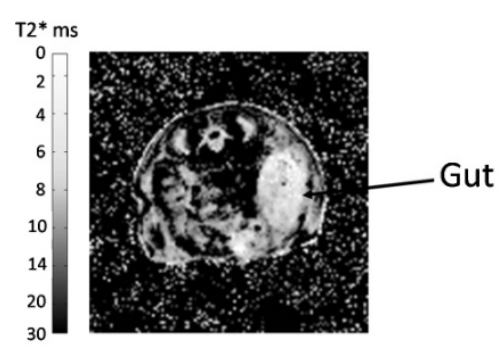

Fig. (5). T2* map of gut. The bright signal correspond to short T2* tissue values. Cortical bones and part of the intestine also present short $\mathrm{T} 2 *$ not evaluated in this work. The intensity scale reports $\mathrm{T} 2 *$ value from 0 to $30 \mathrm{~ms}$.

Fig. (6A) shows stomach and subcutaneous fat under the skin. The signal from the liver is completely suppressed by the application of the long T2* suppression pulse. Also muscle reported zero signal. No signal is left from spinal cord because the $T_{p}(0.3 \mathrm{~ms})$ used was not short enough to detect the ultra-short bone components.

Fig. (6B), which represents the diagonal-SPRITE reference image without the use of the long $\mathrm{T} 2 *$ suppression pulse, reports signal from all the tissues. No contrast is seen between liver and stomach. Fig. (6A) is noisier than B due to the reduced SNR. This was due to both a reduction in stomach signal intensity after applying the pulse and to the total liver signal suppression.

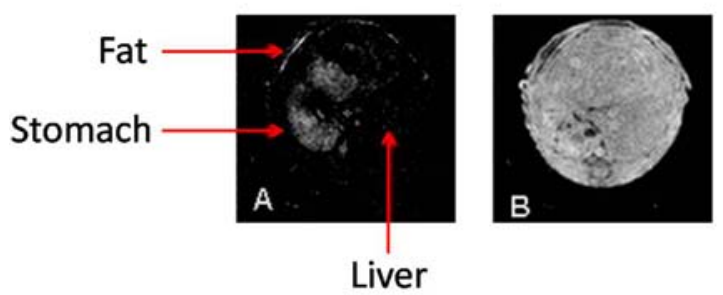

Fig. (6). A) Liver and stomach image after long $\mathrm{T} 2 *$ suppression pulse was applied to the diagonal-SPRITE acquisition. Only stomach is seen as the $\mathrm{T} 2 *$ components from liver was completely suppressed. B) Diagonal-SPRITE reference image of liver and stomach without the use of long $\mathrm{T} 2 *$ suppression pulse.

Fig. (7) reports images from the SPIO-labelled cells in vivo experiment. At the shortest UTE acquisition $\left(\mathrm{T}_{\mathrm{p}}=0.25\right.$ $\mathrm{ms}$ ) the SPIO-labelled cells showed high signal intensity similar to the surrounding tissue and control cells. However, with increasing $T_{p}$ the signal from labelled cells drops significantly leading to negative enhancement. The subtraction between the image acquired at ultra-short $T_{p}(0.25 \mathrm{~ms})$ and the one acquired with a longer $T_{p}(3.5 \mathrm{~ms})$ gives rise to an image with positive contrast in the area where the SPIOlabelled cells were present (Fig. 7F).

The SPIO-labelled cells were confirmed to be in the leg muscle by histological Prussian blue staining (Fig. 8). The mean T2* value of SPIO-labelled cells for the 4 mice was $2.18 \pm 1.07 \mathrm{~ms}$. The control cells, which were injected in the contralateral hind leg, did not show a signal drop at longer $\mathrm{T}_{\mathrm{p}}$ acquisitions and therefore no positive contrast was generated in the subtracted image.

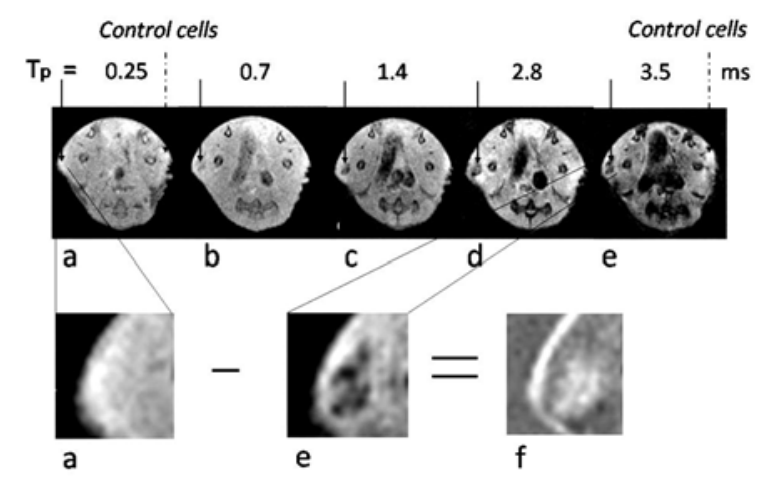

Fig. (7). A-E) Images acquired at different $\mathrm{Tp}$, from an ultra-short $\mathrm{T}_{\mathrm{p}}(0.25 \mathrm{~ms})$ to a short $\left.(3.5 \mathrm{~ms}) ; \mathbf{A}\right)$ area of interest in the hind leg where SPIO-labelled cells were injected; in E) the area drops in signal due to the shortening in $\mathrm{T} 2 * ; \mathbf{F}$ ) subtraction between image $\mathbf{A}$ and $\mathbf{E}$ produces positive contrast that localizes SPIO-labelled cells.

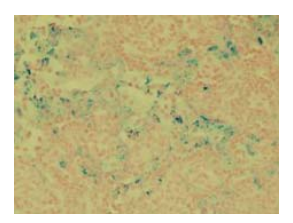

Fig. (8). Photomicrograph showing Prussian blue staining $(\times 400$ magnification) of the hind leg muscles of a C57BL/6 mouse following intramuscular injection of SPIO-labelled cells.

\section{DISCUSSION}

Single point imaging (SPI) developed into SPRITE in order to speed up the acquisition. In this work the raster kspace trajectory has been modified in order to optimize the method for biological models within hardware limitations. A diagonal trajectory was chosen. The diagonal trajectory offers higher spatial resolution images compared to conventional SPRITE technique for the same acquisition time. This is due to the reduced constraint of diagonal-SPRITE on duty cycle and gradient strength.

The diagonal trajectory samples k-space on a Cartesian grid without under-sampling. On the contrary, trajectories proposed in the past years, such as spiral and conicalSPRITE [13], under-sample the frequency space introducing blurring into the image. The trade-off is in acquisition time, conical-SPRITE is faster than diagonal-SPRITE but such sequences are both limited from a duty cycle point of view. The two gradients in the $\mathrm{X}$ and $\mathrm{Y}$ direction, which generally require higher strength to achieve good resolution images, step dynamically through $\mathrm{k}$-space reporting a similar behav- 
iour on gradient temperature rise and consequently on gradient duty cycle.

In diagonal-SPRITE such as in other SPRITE techniques, the acquisition starts from an incoherent steady-state condition avoiding the use of a dummy scan. The incoherent steady-state condition might introduce T1 blurring into the image, but as reported in this paper the use of a small flip angle (around $2^{\circ}$ ) helps to minimize such artefacts. The small flip angle also forces the SPRITE signal to be essentially T2* dependent. Hence, it is appropriate to investigate the $\mathrm{T} 2 *$ blurring which might influence the result image. Because on our $9.4 \mathrm{~T}$ the read-out is as short as $4 \mu \mathrm{s}$ for each single point, the $\mathrm{T} 2 *$ decay is negligible during the acquisition window. Quantitative T2* acquisition can accurately be accomplished.

Improving image quality and spatial resolution while maintaining a short scan time are desirable features for any imaging technique. The scan time for multiple $\mathrm{T}_{\mathrm{p}}$ acquisitions, was minimized by combining diagonal-SPRITE with the multi- $T_{p}$ method. When the $T_{p}$ changes, it is compensated by a variation in gradient amplitude maintaining the same FOV between different $T_{p}$ acquisitions. This method is slower than the multiple $\mathrm{T}_{\mathrm{p}}$ approach introduced by Halse et al. [20] but avoids extra blurring in the images.

The multi- $T_{p}$ used in this work, if compared to single acquisitions, significantly reduced the total detection time of the entire experiment especially in scanner systems, such as ours, where the duty cycle is low. Despite these reductions in acquisition time, the technique remains slow due to the acquisition of the entire FOV. In SPRITE the FOV is a function of the RF coil dimensions, which means that thin slices $(1 \mathrm{~mm}$ ) cannot be acquired in a short acquisition time (a few minutes) using a Cartesian trajectory. An option in further reducing scan time using diagonal-SPRITE might be to reduce the number of acquisition points, for example not detecting the corners of k-space. Pulse preparation might also reduce the acquisition time. For instance a saturation band pulse or a long $\mathrm{T} 2 *$ suppression pulse, positioned in front of each acquisition line, might be useful. A third way to decrease acquisition time is the use of a smaller RF coil in order to reduce the FOV.

One of the potential criticisms of the multi- $\mathrm{T}_{\mathrm{p}}$ diagonalSPRITE is the change in TR when the same k-space line is acquired at different $T_{\mathrm{p}}$. Once again no $\mathrm{T} 1$ effects are introduced in any of the images because the use of a small flip angle makes such artefacts negligible. This gives to the images acquired with a single or a multi- $T_{p}$ method the same quality, in terms of $\mathrm{S} / \mathrm{N}$ and contrast. In addition, the duty cycle limit is not exceeded because in the acquisition the gradients strength is reduced from the ultra-short Tp to the longer $\mathrm{T}_{\mathrm{p}}$.

\section{Applications}

Short T2* values of organs of live normal mice, can be useful to detect baseline information when compared to T2* values of disease models. Similarly baseline data can be compared to oxygen enhancement in pulmonary vessels, or when fibrosis and macromolecular deposition modify areas of the brain and liver. Inflammation of tissues such as ten- dons can be studied with UTE without the use of contrast agents.

The images of normal mouse organs demonstrate high reproducibility, good sensitivity and good resolution; however when the $T_{p}$ time approaches $3 \mathrm{~ms}$ the images start to be noisier. This is due to motion artefacts important at long $\mathrm{T}_{\mathrm{p}}$ acquisitions. To achieve the lowest total scan time and therefore reduce the risk of physiological motion, non gated acquisitions are normally executed. However, respiratory gating or ECG gating needs to be applied when the motion blurs the image to the point that fine details are not distinguishable.

Another approach in highlighting short T2* is given by the pulse suppression experiment. As reported in the result section, liver is completely suppressed leaving visible stomach and fat. Having reported that the short T2* of stomach is of the order of $2.7 \pm 0.3 \mathrm{~ms}$ at $9.4 \mathrm{~T}$, it was reasonable to detect signal from such an organ after the application of a long T2* suppression pulse. However the total absence of liver short $\mathrm{T} 2 *$ components was unexpected. There are several possible explanations. First, mouse liver might only have long $\mathrm{T} 2 *$ components and no ultra-short or short $\mathrm{T} 2 *$, thus validating the suppression. Second, a partial volume effect can cover up small ultra-short T2* tissues. Hardware performance limited spatial resolution, thus the technique could not resolve tissues smaller than the pixel size. Third, the long T2* suppression pulse might not work as efficiently as expected, with some undesirable suppression of short $\mathrm{T} 2 *$ components. Fourth, a magnetization transfer (MT) effect between long and ultra-short $\mathrm{T} 2 *$ components might have reduced the signal intensity of the latter below coil sensitivity.

For the SPIO-labelled cells experiment, no gating was used to limit the scanning time, but details of mouse hind legs are seen. Diagonal-SPRITE collected short T2* which would otherwise have decayed so fast so as to show extremely low signal by standard MRI techniques. In our experiment, it was found that the signal intensity from normal tissue, SPIO-labelled cells and control cells was of similar intensities at ultra-short $\mathrm{T}_{\mathrm{p}}$ values $(0.25 \mathrm{~ms})$. However, at longer $\mathrm{T}_{\mathrm{p}}(3.5 \mathrm{~ms})$ the signal of the labelled and control cells differs, in fact the latter do not drop as dramatically as the signal from SPIO-labelled cells. We took advantage of subtraction (ie subtracting an image with long $\mathrm{T} 2 *$ components from an image with short $\mathrm{T} 2 *$ components) in order to obtain an image with only short $\mathrm{T} 2 *$ components. The subtraction image and the subsequent positive contrast derived from SPIO-labelled cells, helps not only to localize but also to distinguish labelled cells from signal voids and blood. The differentiation is due to the lack of signal detected from voids area at short $T_{p}$ as well as at long $T_{p}$ acquisitions. On the contrary blood would show high signal intensity in a long $\mathrm{T} 2 *$ acquisition.

\section{CONCLUSION}

The preliminary results, reported in this work, show that diagonal-SPRITE provides valuable preclinical in vivo imaging information with improvements in spatial resolution and a reduction in acquisition time when compared to the original SPRITE method. It achieved T2* values of organs, dem- 
onstrated in $\mathrm{T} 2 *$ maps, which are not easily achievable by standard MRI techniques. It shows two ways of highlighting short $\mathrm{T} 2 *$ using subtraction between images at different $\mathrm{T}_{\mathrm{p}} \mathrm{S}$ or adopting a $\mathrm{T} 2 *$ suppression pulse in front of the sequence.

The positive contrast which helps to identify SPIOlabelled cells might be used in the future for all experiments which need to differentiate labelled cells from voids and blood. A positive contrast might also be achieved by the use of a long T2* suppression pulse which was shown to work well on our $9.4 \mathrm{~T}$ scanner. It is worth noting however that although the use of a long $\mathrm{T} 2 *$ suppression pulse reduces the scanning time compared to the subtraction method, it is a more complicated method to implement. Despite this difficulty, RF pulse signal suppression should be considered the method of choice to separate short and long T2* components within the same tissue.

\section{ACKNOWLEDGEMENTS}

The authors would like to acknowledge the use of the Biological Imaging Centre, Imaging Sciences Department, Imperial College London (funded by the Wellcome Trust). We would also thank Manuel Wenten Valenzuela for inspiration.

\section{REFERENCES}

[1] Robson MD, Gatehouse PD, Bydder M, Bydder GM. Magnetic resonance: an introduction to ultrashort TE (UTE) imaging. J Comput Assist Tomogr 2003; 27: 825-46.

[2] Idiyatullin D, Corum C, Park JY, Garwood M. Fast and quiet MRI using a swept radiofrequency. J Magn Reson 2006; 18: 342-49.

[3] Park JY, DelaBarre L, Garwood M. Improved gradient-echo 3D magnetic resonance imaging using pseudo-echoes created by frequency-swept pulses. Magn Reson Med 2006; 55: 848-857.

[4] Tyler DJ, Robson MD, Henkelman RM, Young IR, Bydder GM. Magnetic resonance imaging with ultrashort TE (UTE) PULSE sequences: Technical considerations. J Magn Reson Imaging 2007; 25: 279-89.

[5] Rahmer J, Bornert P, Groen J, Bos C. Three-dimensional radial ultrashort echo-time imaging with T2 adapted sampling. Magn Reson Med 2006; 55: 1075-82.
[6] Bergin C, Pauly J, Macovski A. Lung Perenchyma: projection Reconstruction MR Imaging. Radiology 1991; 179: 777-781.

[7] Schmidt M, Yang G, Gatehouse P, Firmin D. FID-Based Lung MRI at $0.5 \mathrm{~T}$ : Theorietical Considerations and Pratical Implications. Magn Reson Med 1998; 39: 666-72.

[8] Gold G, Pauly J, Glover G, Moretto J, Macoviski A, Herkens R. Characterization of Atherosclerosis with a 1.5T Imaging System. J Magn Reson Imaging 1993; 3: 399-407.

[9] Glover G, Pauly J, Bradshaw K. Boron-11 Imaging with 3dimensional Reconstruction method. J Magn Reson Imaging 1992; 2: 47-52.

[10] Reichert IL, Robson MD, Gatehouse PD, et al. Magnetic resonance imaging of cortical bone with ultrashort TE pulse sequences. J Magn Reson Imaging 2005; 23: 611-18.

[11] Gravina S, Cory DG. Sensitivity and Resolution of Constant-time Imaging. J Magn Reson 1994; Serie B: 53-61.

[12] Balcom BJ, Macgregor RP, Beyea SD, Green DP, Armstrong RL, Bremner TW. Single-Point Ramped Imaging with T1 Enhancement (SPRITE). J Magn Reson A 1996; 123: 131-34.

[13] Halse M, Goodyear DJ, MacMillan B, Szomolanyi P, Matheson D, Balcom BJ. Centric scan SPRITE magnetic resonance imaging. J Magn Reson 2003; 165: 219-29.

[14] Marble AE, Mastikhin IV, MacGregor RP, et al. Distortion-free single point imaging of multi-layered composite sandwich panel structures. J Magn Reson 2004; 168: 164-74.

[15] Romanzetti S, Halse M, Kaffanke J, Zilles K, Balcom BJ, Shah NJ. A comparison of three SPRITE techniques for the quantitative 3D imaging of the (23) Na spin density on a $4 \mathrm{~T}$ whole-body machine. $\mathrm{J}$ Magn Reson 2006; 179: 64-72.

[16] Olsson LE, Lindahl M, Onnervik PO, et al. Measurement of MR signal and $\mathrm{T} 2 *$ in lung to characterize a tight skin mouse model of emphysema using single-point imaging. J Magn Reson Imaging 2007; 25: 488-94.

[17] Sharp JC, Bowtell RW, Mansfield P. Elimination of susceptibility distortion and reduction of diffusion attenuation in NMR microscopy by line-narrowed 2DFT. Magn Reson Med 1993; 29: 407-11.

[18] Mastikhin IV, Balcom BJ, Prado PJ, Kennedy CB. SPRITE MRI with prepared magnetization and centric k-space sampling. J Magn Reson 1999; 136: 159-68.

[19] Larson PE, Gurney PT, Nayak K, Gold GE, Pauly JM, Nishimura DG. Designing long-T2 suppression pulses for ultrashort echo time imaging. Magn Reson Med 2006; 56: 94-103.

[20] Halse M, Rioux J, Romanzetti S, et al. Centric scan SPRITE magnetic resonance imaging: optimization of SNR, resolution, and relaxation time mapping. J Magn Reson 2004; 169: 102-17.

(C) Protti et al.; Licensee Bentham Open.

This is an open access article licensed under the terms of the Creative Commons Attribution Non-Commercial License (http://creativecommons.org/licenses/by-nc/3.0/) which permits unrestricted, non-commercial use, distribution and reproduction in any medium, provided the work is properly cited. 